\title{
Decentralized Coordinated Control of Multi-infeed HVDC System for Damping Inter-area Oscillation
}

\author{
FAN YANG, CHEN CHEN, XITIAN WANG \\ Department of Electrical Engineering \\ Shanghai Jiaotong University \\ No. 800, DongChuan road, Shanghai \\ CHINA
}

\begin{abstract}
An important concern in the operation of multi-infeed HVDC systems is the inter-area low frequency oscillation due to the long-distance bulk-power transmission. In this paper, a decentralized coordinated control design is presented for improvement of inter-area oscillations in the multi-infeed HVDC system. A DC modulation control low is designed based on LQR optimal method and decentralized observers. Without degradation of controller performance, only the local measurement output is required for each modulation controller, which greatly reduces the communication burden. A 7-generator, 2-HVDC system was used as the test system. The eigenvalue analysis and nonlinear time domain simulation have demonstrated the validity of the proposed decentralized controllers.
\end{abstract}

Key Words: -Multi-infeed HVDC, Low frequency oscillation, Decentralized control, HVDC modulation control, Linear-quadratic regulator, State observer

\section{Introduction}

With the development of China's power industry, High Voltage DC transmission technology has been widely used for long-distance bulk-power delivery and asynchronous interconnections. For the next few decades, more HVDC transmission projects will be constructed in China to meet the requirement of power transmission from the energy centers to the load centers [1]. As a result of the growing use of HVDC technology, there are two or more HVDC links embedded in the same synchronous grid and feeding the same electrical area. In such a large-scale multi-regional interconnected $\mathrm{AC} / \mathrm{DC}$ power grid, inter-area low-frequency oscillation is usually a problem.

HVDC modulation is an effective approach to suppress the inter-area low-frequency oscillation for its fast and huge adjustable power [2]. However, improper control coordination between multiple HVDC links may weaken the effect of damping modulations and lead to undesirable interactions [3]. In recent years, considerable efforts have been made to explore the coordinated control of multiple HVDC links [4-6]. Most of the researches have concentrated on simulation method, which require substantial experiences and repetitive trial by error. Since finding the coordinated control law of multiple HVDC links is not an easy task, it is important to establish a systemic design procedure.
Linear-Quadratic Regulator (LQR) optimal control method has been widely used in the design of the power system controllers. The performance object is a quadratic function of state vectors and control inputs. The optimal control law can be obtained by solving the algebraic Riccati equation. One problem to implement such a design method is that it requires full state feedback. To overcome the problems, an alternative approach is to use the decentralized observers to constructs the system states for each feedback channel [7-8].

In this paper, a decentralized coordinated control scheme is designed to damp inter-area oscillations in the multi-infeed HVDC system. A system model containing seven generators and two HVDC links was used as the test system. First, the low frequency oscillation analysis is carried out based on the eigen-analysis method. Then, the two HVDC modulation controllers are designed based on LQR technology and decentralized observers. Without degradation of controller performance, only the adjacent AC tie-line's active power is required for each modulation controller respectively, which greatly reduces the communication burden. Eigenvalue and simulation results using the proposed controllers are presented. The results demonstrate that the decentralized coordinated modulation controllers can damped the inter-area oscillations effectively. 


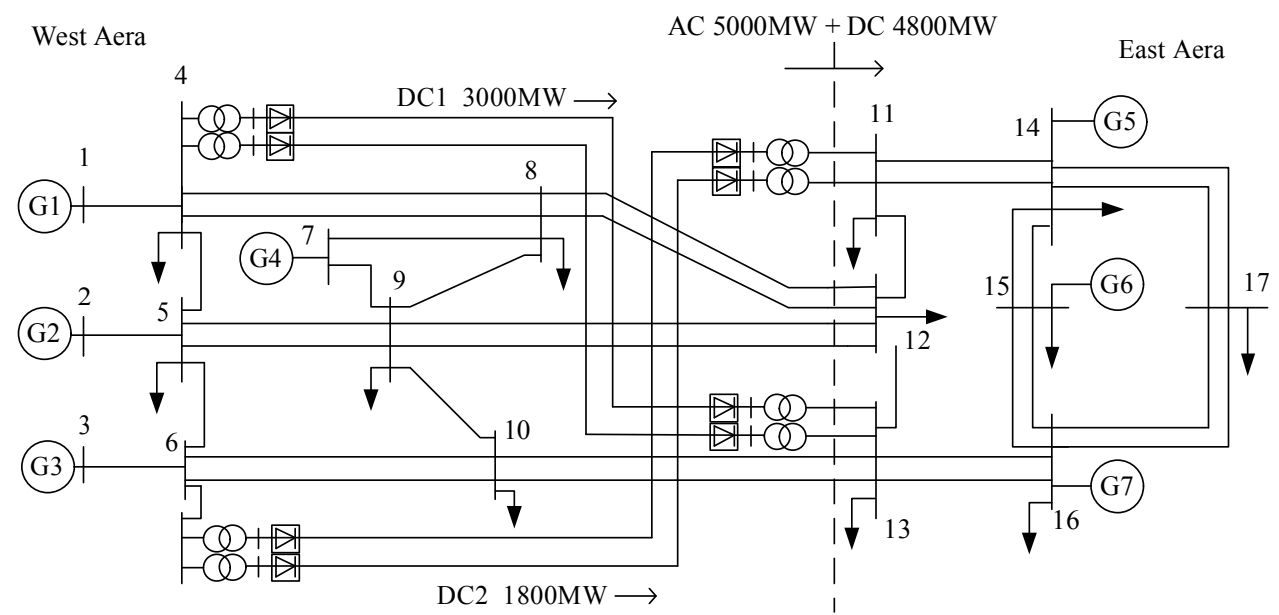

Fig.1 Configuration diagram of the multi-infeed AC/DC power system

\section{System Model and Oscillation Damping Analysis}

A typical AC/DC hybrid system under consideration is shown in Fig.1. The system studied is based on a simplified model of China South Grid, which contains 7 equivalent generators, 17 buses, 12 loads. As shown in Fig. 1, two HVDC links and three AC transmission corridors deliver large amounts power from the west area to the east area. DC1 represents a bipolar link with a voltage rating of $\pm 500 \mathrm{kV}$ and a power rating of $3000 \mathrm{MW}$. DC2 has similar parameters of $\pm 500 \mathrm{kV}$ and $1800 \mathrm{MW}$.

For small signal analysis of low frequency oscillation, all the generators are modelled in detail. Each generator is equipped with third order excitation control system. It is assumed that the mechanical power input of each generator is constant. The loads are represented as constant MVA model. The AC networks are modelled by algebraic equations, therefore representing only the fundamental frequency behavior of the system. Detailed HVDC modelling is adopted, in which the DC converters are modelled by quasi-steady equations. The DC line is modelled by differential equations as a $\mathrm{T}$ circuit, which includes the resistance and inductance of smoothing inductors. In normal operation, the rectifier controller is in constant current mode and the inverter controller is in constant extinction angle mode by PI control.

The eigenvalues of the overall system were calculated at the equilibrium operation point. This resulted in 75 eigenvalues. 6 electromechanical modes of oscillation are presented in Table 1. Fig. 2 shows the mode shapes of the two weak-damped modes, mode 1 at $0.75 \mathrm{~Hz}$ and mode 2 at $0.85 \mathrm{~Hz}$.
Table 1 Electromechanical modes of the system

\begin{tabular}{|c|c|c|c|}
\hline Mode & Eigenvalue & $\begin{array}{c}\text { Frequency } \\
(\mathrm{Hz})\end{array}$ & $\begin{array}{c}\text { Damping } \\
\text { ratio(\%) }\end{array}$ \\
\hline 1 & $-0.0523 \pm j 4.709$ & 0.75 & 1.11 \\
\hline 2 & $-0.1073 \pm j 5.364$ & 0.85 & 2.00 \\
\hline 3 & $-0.3018 \pm j 6.361$ & 1.01 & 4.74 \\
\hline 4 & $-0.4690 \pm j 7.276$ & 1.16 & 6.43 \\
\hline 5 & $-0.8392 \pm j 8.164$ & 1.30 & 10.23 \\
\hline 6 & $-0.8832 \pm j 8.187$ & 1.30 & 10.73 \\
\hline
\end{tabular}

Further analysis of mode shape indicates that the first two weak-damped modes, mode 1 and mode 2, belong to the inter-area modes, as shown in Fig.2. The other four modes are local modes. Mode 1 represents the generator G1 swinging against G3, G5, G6 and G7. Mode 2 represents G2, G3 swinging against G1, G5, G6, G7. Mode 3 and mode 4 reflects the swing of generators within the west area. Mode 5 and mode 6 reflects the relative swing within the east area. This paper concentrates on the suppression of the two weak-damped inter-area modes because of their importance to the overall stability of the system.

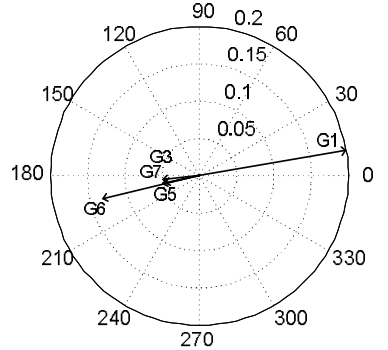

(a) Mode 1

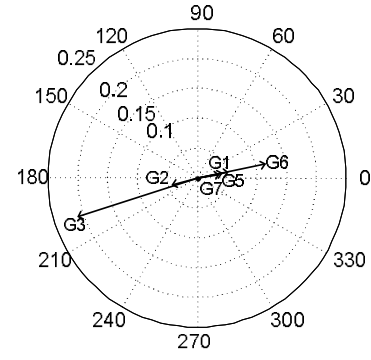

(b) Mode 2
Fig.2 Mode shape scatter diagram of Mode 1 and Mode 2 
In order to improve the damping of the inter-area modes in the system, both DC1 and DC2 are configured with modulation controllers by adding an additional signal to the rectifier power reference. The controllability analysis shows that, the modulation controllers at the two rectifiers have considerable effects on the inter-area modes but poor control effects on the local modes.

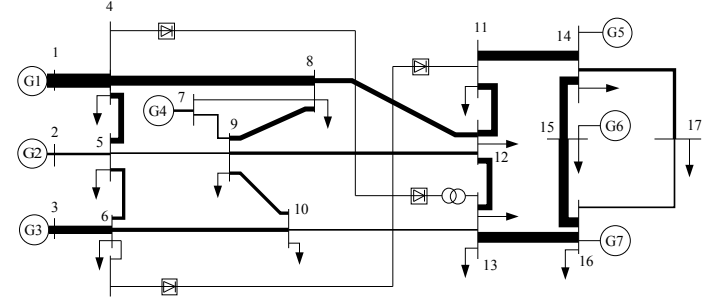

(a) Mode 1

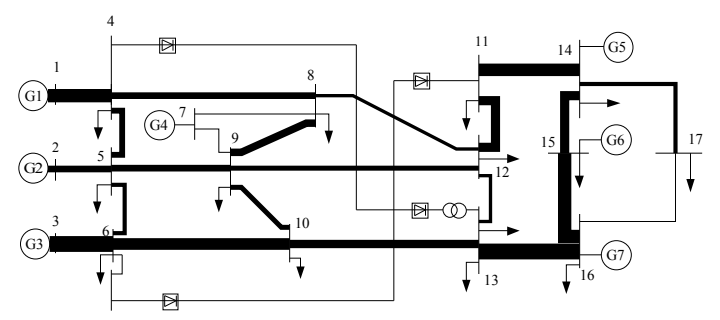

(b) Mode 2

Fig. 3 Observability diagram of mode 1 and mode 2

The power deviations in the AC lines are selected as the candidates of feedback signals. The observability analysis of each AC line is carried out. The results for mode 1 and mode 2 are illustrated in Fig.3, in which the width of the AC lines is proportional to the degree of its observability. It can be seen from Fig. 3 that, line 4-8 has preferable observability for mode 1 , while line 6-10 has better observability for mode 2 . Therefore, the structure of the decentralized modulation controller is determined by (1) as follows

$$
\left[\begin{array}{l}
\Delta P_{\bmod 1} \\
\Delta P_{\bmod 2}
\end{array}\right]=\left[\begin{array}{ll}
H_{1}(s) & \\
& H_{2}(s)
\end{array}\right]\left[\begin{array}{c}
\Delta P_{4-8} \\
\Delta P_{6-10}
\end{array}\right]
$$

\section{Design of LQR Optimal Control Based on Decentralized Observers}

For the design of HVDC modulation controller, the linearized state-space equations of the open loop system can be expressed in block form as

$$
\left\{\begin{array}{l}
\dot{\mathbf{X}}=\mathbf{A X}+\mathbf{B U} \\
\mathbf{Y}=\mathbf{C X}
\end{array}\right.
$$

where $\mathbf{X}$ is state vector of the system and $\mathbf{U}=$ $\left[\Delta P_{\bmod 1}, \Delta P_{\bmod 2}\right]^{\mathrm{T}}, \mathbf{Y}=\left[\Delta P_{4-8,}, \Delta P_{6-10}\right]^{\mathrm{T}}$.
The decentralized modulation controller design is based on LQR optimal control theory. A performance index $\mathrm{J}$ can be selected as

$$
J=\int_{0}^{\infty}\left[\mathbf{X}^{\mathrm{T}} \mathbf{Q} \mathbf{X}+\mathbf{U}^{\mathrm{T}} \mathbf{R} \mathbf{U}\right] d t
$$

where $\mathbf{Q}$ and $\mathbf{R}$ are symmetric weight matrices, and they are positive semi-definite and positive definite respectively. To minimize the performance index, $\mathbf{Q}$ and $\mathbf{R}$ should be appropriately designed. Then the optimal control law is obtained as

$$
\begin{aligned}
\mathbf{U} & =-\mathbf{R}^{-1} \mathbf{B}^{\mathrm{T}} \mathbf{P X} \\
& =-\mathbf{K X}
\end{aligned}
$$

where $\mathbf{K}$ is the gain matrix of full state feedbacks. $\mathbf{P}$ is the positive definite, symmetric matrix to satisfy the algebraic Riccati equation

$$
-\mathbf{P A}-\mathbf{A}^{\mathrm{T}} \mathbf{P}+\mathbf{P B R}^{-1} \mathbf{B}^{\mathrm{T}} \mathbf{P}-\mathbf{Q}=\mathbf{0}
$$

Actually, the full states of the system cannot be obtained by direct measure. Since the system is observable, it is possible to fully reconstruct the system states from its output measurements using the state observer, as shown in Fig.4.

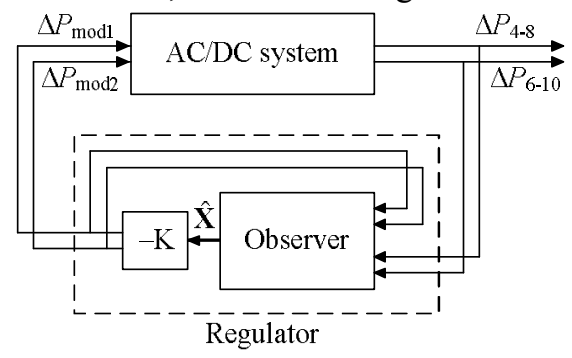

Fig.4 LQR control based on centralized observer

This traditional observer requires additional communication channel to transfer the remote measurements, $\Delta \mathrm{P} 4-8$ and $\Delta \mathrm{P} 6-10$, to the observer. However, according to (1), the decentralized control requires that the $\mathrm{DC} 1$ controller uses only the output $\triangle \mathrm{P} 4-8$ which is measured at the adjacent tie-line to compute the control signal $\triangle \mathrm{Pmod} 1$, and DC2 uses only the output $\Delta \mathrm{P} 6-10$ to compute the control signal $\Delta \mathrm{Pmod} 2$.

In order to overcome the problem, a feasible approach is to use the decentralized observers to constructs the system states for each feedback channel. The basic idea is that the system states can be reconstructed at different location where measurements are available. Then, the state feedback can be implemented locally to achieve the LQR control performance. The decentralized control framework is shown in Fig.5. For the decentralized control, the remote measurements are not required and the local measurements are sufficient. The dynamic performance of decentralized control can be made nearly equal to that of centralized control, if the state observers are designed appropriately. 


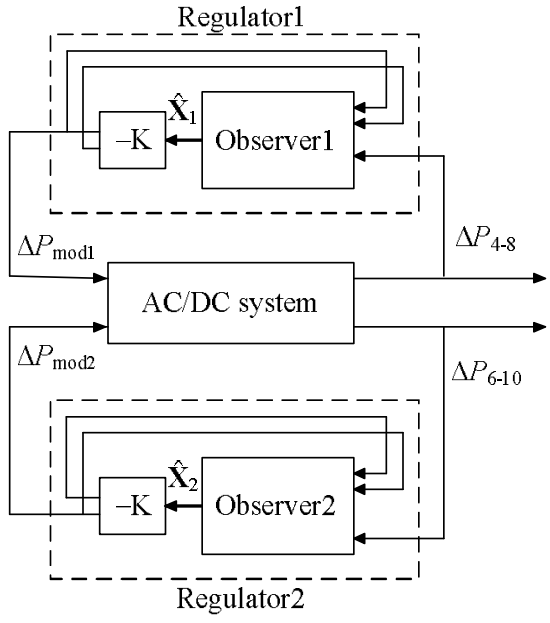

Fig.5 LQR control based on decentralized observer

It should be noted that, the controllers using the design scheme have the same orders of the AC/DC power systems. The controllers of such a high order (75 in this system) are undesired and hard to be implemented. There are usually two ways to solve this problem. One way is to reduce the system first and then design the controllers. The other way is to design the controllers first and reduce its order. In this paper, the order of the two controllers can be reduced to 8 , while the dynamic performance is comparable to that of the full order controllers.

Table 2 Electromechanical modes with the proposed controllers

\begin{tabular}{|c|c|c|c|}
\hline Mode & Eigenvalue & $\begin{array}{c}\text { Frequency } \\
(\mathrm{Hz})\end{array}$ & $\begin{array}{c}\text { Damping } \\
\text { ratio(\%) }\end{array}$ \\
\hline 1 & $-0.5766 \pm j 4.884 \mathrm{i}$ & 0.78 & 11.7 \\
\hline 2 & $-0.3919 \pm j 5.381 \mathrm{i}$ & 0.86 & 7.26 \\
\hline 3 & $-0.3431 \pm j 6.358 \mathrm{i}$ & 1.01 & 5.39 \\
\hline 4 & $-0.4733 \pm j 7.279 \mathrm{i}$ & 1.16 & 6.49 \\
\hline 5 & $-0.8421 \pm j 8.161 \mathrm{i}$ & 1.30 & 10.26 \\
\hline 6 & $-0.8837 \pm j 8.186 \mathrm{i}$ & 1.30 & 10.73 \\
\hline
\end{tabular}

The eigenvalues of the closed loop system with the proposed HVDC modulation controllers are given in Table 2. Compared with Table 1, the damping ratio of the weak-damped modes has been improved effectively. The damping ratio of mode 1 and mode 2 rises to $11.7 \%$ and $7.26 \%$ respectively. It seems that the HVDC modulation controllers only slightly improve the local modes, which is consistent with the results of the controllability analysis above.

\section{SIMULATION RESULTS}

To verify the validity of the designed controllers, the system shown in Fig. 1 is simulated by the transient simulation software PSCAD/EMTDC. The HVDC converters were represented by the in-built
EMTDC model. The AC filters and transformers on either side of the HVDC links are represented in detail.

In the simulation, two disturbance cases are tested. One is the three phase-ground fault occurs at Bus 12 cleared after five cycles (about $0.10 \mathrm{~s}$ ). The other is the line tripping fault happens to one of the parallel line 5-9. In the first case, the disturbance is relatively slight. Hence, the pre-fault equilibrium point is nearly same to the post-fault equilibrium point. In the second case, the topology of the network is changed, the post-fault equilibrium point drifts away from the pre-fault equilibrium point. The robustness of the proposed decentralized controllers is evaluated by this case.

The system responses with and without the decentralized modulation control are shown in Fig. 6 and Fig. 7. It can be observed that, without modulation control, there exists sustained power oscillation and generator-angle oscillation after the faults. Although only the local measurements are required, the decentralized controllers can damp the oscillation effectively, and it is robust to the large disturbance.

From these time domain simulation results, it can be seen that the proposed decentralized controllers based on the decentralized observer is effective in damping the low-frequency oscillation after small or large disturbance. The eigenvalue analysis and nonlinear time domain simulation have demonstrated the validity of the proposed decentralized controllers.
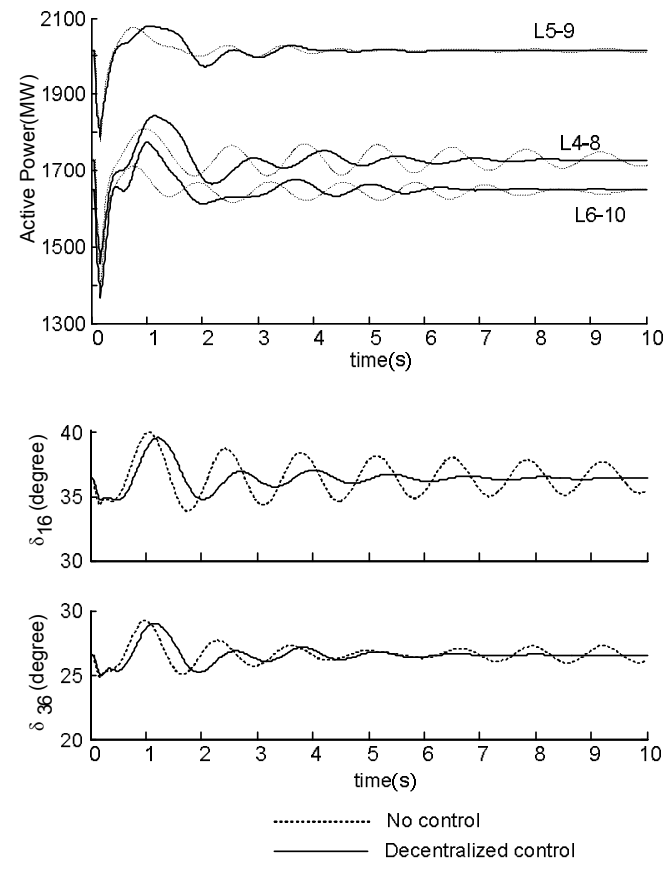

Fig.6 Response for three phase-ground fault 

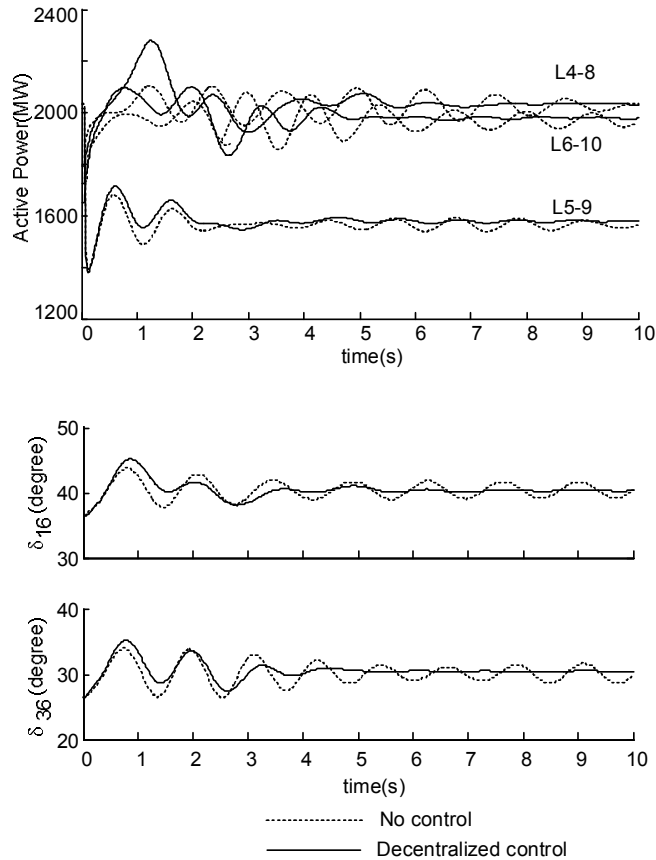

Fig.7 Response for line tripping fault

\section{Conclusions}

In this paper, a decentralized coordinated control scheme is applied for the improvement of inter-area oscillations in the multi-infeed HVDC system. The coordination design is based on LQR optimal control method and decentralized observers. In order to reduce the communication burden, the system states are restructured at different locations by using the local measurement output only. Then, the full state feedback can be implemented locally to achieve the LQR control performance. A 7-generator, 2-HVDC system was used as the test system. The eigenvalue analysis and nonlinear time domain simulation have demonstrated the validity of the proposed decentralized controllers.

In addition, this decentralized control scheme may also be applicable to other coordination control problems in power system, such as the coordination control of HVDC and FACTS devices.

\section{Acknowledgment}

This study is supported by the National Natural Science Foundation of China (No. 50595410).

\section{References:}

[1] Ying Huang, Zheng Xu. "Study on the pure DC transmission scheme for China's future power transmission from the West to the East," Power Engineering Society General Meeting, 2004 IEEE, June 2004, Vol. 2, pp. 1459-1463

[2] Kundur P, Power System Stability and Control, New York: McGraw-Hill, 1994.

[3] L.A.S. Pilotto, M. Szechtman, A. Wey, W.F. Long et al. "Synchronizing and damping torque modulation controllers," IEEE Trans. on Power Delivery, Vol. 10, no. 3, pp. 1505-1513, 1995

[4] Haifeng Liu, Zheng $\mathrm{Xu}$, Licheng Jin, "Coordination and optimization of small signal modulators in multi-infeed HVDC systems," Transmission and Distribution Conference and Exposition, 2003 IEEE PES, Sept. 2003, Vol. 1, pp. $35-40$

[5] Mao Xiaoming, Zhang Yao, Guan Lin, Wu Xiaochen. "Researches on coordinated control strategy for inter-area oscillations in AC/DC hybrid grid with multi-infeed HVDC," Transmission and Distribution Conference and Exhibition: Asia and Pacific, 2005 IEEE/PES, pp. $1-5$

[6] Chao Lu, Jennie Si, Xiaochen $\mathrm{Wu}$, Peng Li, "Approximate Dynamic Programming Coordinated Control in Multi-infeed HVDC Power System," Power Systems Conference and Exposition, PSCE '06. 2006 IEEE PES, pp. 2131-2135

[7] C.E. Grund. "Unified Active and Reactive Power Modulation of HVDC systems: Large-Signal and Distributed Small-Signal Control," EPRI Research Project, EPRI-EL-4822, Oct. 1986

[8] Seung-Cheol Lee, Seung-Il Moon, Jang-Cheol Seo, Jong-Keun Park, "Observer-Based Decentralized Optimal Controller Design of PSS and TCSC for Enhancement of Power System Dynamic Stability," PES Summer Meeting, 2000, IEEE, July 2000, Vol. 3, pp. 1942-1945 